\title{
Comparação de dados dos satélites Ikonos-II e Landsat/ETM+ no estudo de áreas cafeeiras
}

\author{
Gláucia Miranda Ramirez(1), Jurandir Zullo Junior(2), Eduardo Delgado Assad(3) e Hilton Silveira Pinto(2)
}

\begin{abstract}
(1)Universidade Estadual de Campinas (Unicamp), Fac. de Engenharia Agrícola, Planejamento e Desenvolvimento Rural Sustentável, Caixa Postal 6.011, CEP 13083-875 Campinas, SP. E-mail: glaucia@cpa.unicamp.br (2)Unicamp, Centro de Pesquisas Meteorológicas e Climáticas Aplicadas à Agricultura, Cidade Universitária Zeferino Vaz, CEP 13083-970 Campinas, SP. E-mail: jurandir@cpa.unicamp.br, hilton@cpa.unicamp.br (3)Embrapa Informática Agropecuária, Caixa Postal 6.041, CEP 13086-970 Campinas, SP. E-mail: assad@cnptia.embrapa.br
\end{abstract}

Resumo - O objetivo deste trabalho foi avaliar o impacto do aumento da resolução espacial e radiométrica da imagem pancromática do Ikonos-II na identificação de plantios de café (Coffea arabica), em comparação com as imagens do Landsat/ETM+. A área de estudo está localizada no Município de Pedregulho, SP, onde foram selecionados 50 talhões com plantios de café, e foram levantados dados referentes à altura, idade, espaçamento e variedade de cada talhão. As imagens permitiram a identificação de talhões com características diferentes em campo, tendo-se destacado a imagem do Ikonos-II, que apresentou melhor desempenho. Para os talhões com características iguais em campo, as imagens analisadas não se mostraram eficientes, independentemente do satélite utilizado. As correções atmosféricas e radiométricas, na imagem do Ikonos-II, não proporcionaram ganho efetivo nas análises realizadas. A maioria dos talhões identificados na imagem do Ikonos-II pode ser localizada na imagem do Landsat/ETM+ (68\%). A correlação significativa entre a banda 4 do Landsat/ETM+ e o canal pancromático do Ikonos-II indica uma forma de ligação entre as imagens dos dois satélites.

Termos para indexação: Coffea arabica, sensoriamento remoto, imagem pancromática.

\section{Comparison between Ikonos-II and Landsat/ETM+ satellites data in the study of coffee areas}

\begin{abstract}
The objective of this work was to assess the impact of the better spatial and radiometric resolutions of the Ikonos-II panchromatic image, for the identification of coffee (Coffea arabica) planting areas, in comparison with a Landsat/ETM+ image. The area of study is situated in the city of Pedregulho, State of São Paulo, Brazil, where 50 coffee fields were selected. Information about plants features, like height, age, spacing and variety were collected. Images allowed the identification of coffee areas with different field features, and the calibrated Ikonos-II image showed the best results. Considering the areas with similar field features, images from both satellites were not efficient in the coffee identification. The atmospheric and radiometric corrections applied on the Ikonos-II image did not improve the analyses results. More than half of the identified areas in the Ikonos-II image could be found in the Landsat/ETM+ image (68\%). The significant correlation between Landsat/ETM+ band 4 and Ikonos-II panchromatic channel shows a link between both satellite images.
\end{abstract}

Index terms: Coffea arabica, remote sensing, panchromatic image.

\section{Introdução}

Estimativas da produção agrícola são de grande importância para a economia do país, uma vez que fornecem suporte aos formuladores de políticas agrícolas e aos tomadores de decisão do setor privado, ligados a atividades agropecuárias (Vicente et al., 1990). Para commodities agrícolas, como o caso do café, informações precisas, em tempo hábil e com baixo custo, sobre a extensão e o rendimento da cultura são instrumentos valiosos para o planejamento e comercialização das safras. Tendo-se em vista que produção de café é um importante segmento da economia brasileira, a implementação de previsão de safra confiável pode oferecer um planejamento adequado, em virtude da correta integração entre o mercado e a produção (Liu \& Liu, 1988).

No Brasil, apesar da reconhecida importância de se determinar os níveis de produção e a extensão ocupada 
por culturas agrícolas, a metodologia operacional é subjetiva e não dispõe de métodos que possam fornecer uma espacialização da área com as culturas. Por essa razão, é imprescindível o desenvolvimento de metodologias objetivas, que permitam obter informações da área, bem como da produção, em curto espaço de tempo, a menor custo e com confiabilidade conhecida.

O uso do sensoriamento remoto orbital e das técnicas de geoprocessamento representa um avanço significativo na obtenção de informações sobre dados da agricultura, por seu caráter multiespectral, sua repetividade, seu custo e por ser uma metodologia menos sujeita a erros de análise humana (Dallemand et al., 1988; Moreira, 1991; Epiphanio et al., 1994). A utilização crescente dessas imagens, ao longo de mais de duas décadas, tem permitido a obtenção de resultados positivos, em prazos adequados às necessidades dos usuários, e com maior precisão e menor custo, quando comparada com técnicas convencionais (Gonzalez-Alonso et al., 1997; Ippoliti-Ramilo et al., 1999).

Os satélites utilizados para avaliação dos recursos naturais sofreram sensíveis mudanças tecnológicas nos sensores, ao longo do tempo, principalmente no que se refere às resoluções espacial e espectral. Essas mudanças tecnológicas permitiram desenvolver sensores de alta resolução espacial, como é o caso do Ikonos-II e do Quickbird, que pode chegar a centímetros. Houve, evidentemente, melhoria na resolução radiométrica, em comparação a sensores como o ETM+, do Landsat-5, entre outros. Esse avanço tecnológico permitiu identificar e mapear talhões de culturas de difícil separabilidade, até então, nos dados coletados por sensores de média resolução.

No contexto da produtividade, há vários trabalhos desenvolvidos com dados do Landsat (Batista et al., 1990; Hamar et al., 1995; Epiphanio et al., 1996; Fang et al., 1998; Lunetta et al., 2002; Ippoliti-Ramilo et al., 2003; Lobell \& Asner, 2003). Acredita-se que os métodos empregados para obter dados relativos à produtividade poderão ser substancialmente melhorados, pela utilização de dados com melhor resolução espacial e radiométrica.

Trabalhos que objetivaram avaliar o potencial do uso de imagens do satélite Ikonos-II, no planejamento urbano e ambiental, têm mostrado os benefícios advindos de sua melhor resolução espacial (Song \& Woodcock, 2002; Sugumaran et al., 2002; Herold et al., 2003; Lee et al., 2003; Read et al., 2003).
No âmbito agrícola, estas imagens têm sido utilizadas para diversos fins, desde o levantamento do uso do solo até a determinação de índices de área foliar, com base em índices de vegetação. Dechka et al. (2002), Colombo et al. (2003), Johnson (2003) e Johnson et al. (2003) mostraram as vantagens da associação da alta resolução espacial com a melhoria da resolução radiométrica, nas imagens do satélite Ikonos-II, para fins agrícolas.

$\mathrm{O}$ aprimoramento das resoluções espacial e radiométrica, nas imagens de sensoriamento remoto, aliado ao desenvolvimento das técnicas de geoprocessamento, podem proporcionar benefícios na identificação de plantios de café, visando a mensurar e monitorar as áreas plantadas com esta cultura, permitindo previsões de safra com maior confiabilidade que o atual.

O objetivo deste trabalho foi avaliar o ganho da resolução espacial e radiométrica de imagens do Ikonos-II, na identificação de plantios de café (Coffea arabica), em comparação com as imagens do Landsat/ETM+.

\section{Material e Métodos}

A área de estudo localiza-se na Fazenda Taquari, a $20^{\circ} 10^{\prime} \mathrm{S}, 47^{\circ} 24^{\prime} \mathrm{W}$ e $20^{\circ} 12^{\prime} \mathrm{S}, 47^{\circ} 22^{\prime} \mathrm{W}$, no Município de Pedregulho $\left(47^{\circ} 23^{\prime} \mathrm{W}\right.$ e $\left.20^{\circ} 12^{\prime} \mathrm{S}\right)$, SP, em uma das regiões mais tradicionais na produção de café no Brasil, a Mogiana. Segundo Picini et al. (1999), a Mogiana possui o agrossistema mais produtivo e sustentável de São Paulo, em virtude de apresentar solos mais adequados, maior adoção de tecnologia e renovação do parque cafeeiro, melhor qualidade de bebida e, fundamentalmente, melhores condições climáticas para cultivo do café Arábica.

Foram selecionados, dentro da propriedade, 50 talhões de café, com diferentes áreas, alturas de planta, datas de plantio (idade), variedades e espaçamentos parâmetros pesquisados em campo, para cada talhão.

A altura foi agrupada em quatro classes, com diferença de $1 \mathrm{~m}$ entre classes, com o limite inferior e superior obtidos de acordo com a menor e maior altura das plantas encontradas nos talhões analisados. A idade também foi agrupada em quatro classes, com diferença de um ano entre as classes, e os espaçamentos foram agrupados em duas classes, considerando-se, para tanto, somente o espaçamento entre linhas. Nos talhões avaliados foram encontradas as variedades Mundo Novo, Catuaí, Icatu, Iapar, Obatã e Acaiá. 
Foram empregadas imagens dos satélites Ikonos-II e Landsat/ETM+, para obtenção das informações espectrais desses talhões. A imagem do canal pancromático do Ikonos-II foi utilizada, com resolução espacial de $1 \mathrm{~m}^{2}$, em que foi adotado como datum de referência, o WGS-84, zona 23S. A imagem foi adquirida em 29/9/2000, com ângulo azimutal solar e do satélite de 64,32 e $86,6^{\circ}$, respectivamente. O ângulo de elevação solar foi de $54,85^{\circ}$ e o do satélite, $50,6^{\circ}$ (Figura $1 \mathrm{~A}$ ).

A imagem do satélite Landsat/ETM+ foi obtida em 28/9/2000, correspondente à órbita/ponto 220/074, com resolução espacial de 30x30 m (900 m²). Foram utilizadas as imagens das bandas 3, 4 e 5 do sensor ETM+. As duas imagens foram adquiridas quando os cafezais estavam no estádio de florescimento (Figura 1 B).

Na correção radiométrica e atmosférica, foi utilizado o sistema computacional Scoradis (Sistema de Corre- ção Radiométrica de Imagens de Satélite), desenvolvido por Zullo Junior (1994), que se baseia na teoria e parâmetros atmosféricos calculados pelo Modelo 5S (Tanré et al., 1990). Posteriormente, foi realizada a coleta dos valores digitais da imagem pancromática do satélite Ikonos-II . Os valores de reflectância real, das áreas cafeeiras, foram coletados tanto na imagem pancromática do Ikonos-II, como nas imagens das bandas 3, 4 e 5 do Landsat/ETM+; os sistemas de tratamento de imagens digitais ENVI 3.4 e 3.5 foram utilizados para a extração desses valores.

Os 50 talhões levantados em campo foram identificados e selecionados, primeiramente, na imagem do Ikonos-II. A área útil dentro de cada talhão, para adquirir os valores de reflectância, foi total, eliminando-se apenas os pixels de borda, para evitar a contaminação da reflectância dos alvos vizinhos. Assim, não ocorreu amostragem de pixels, mas sim a seleção de todos os pixels que compunham cada talhão.

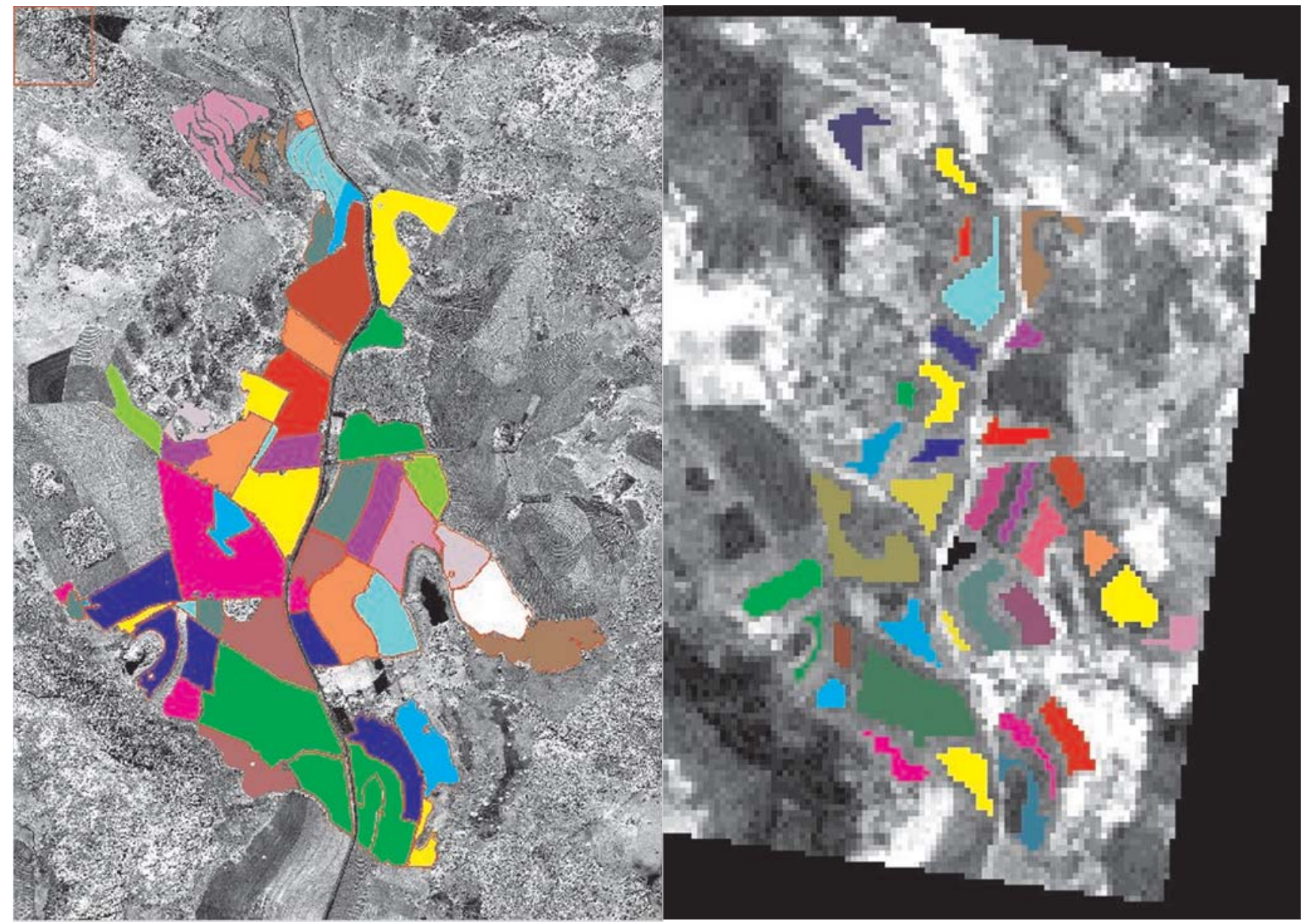

Figura 1. Imagens pancromáticas dos satélites Ikonos-II (A) e Landsat/ETM+ (B), mostrando os talhões que foram identificados. 
Para selecionar os talhões de maneira automática, na imagem do Landsat/ETM+, foi necessário o georreferenciamento da imagem com base na do IkonosII. A partir da escolha da imagem base - a do Ikonos-II, que fornece as informações geográficas - a imagem do Landsat/ETM+ era corrigida. Com a localização exata dos talhões, na imagem Landsat/ETM+, foi realizada a exclusão dos pixels externos (1 pixel de toda a borda), evitando-se, assim, a contaminação pelos pixels vizinhos. Portanto, para análise da imagem Landsat/ETM+, foram considerados os talhões que, após a eliminação da borda, continham, no mínimo, 10 pixels.

O método utilizado neste trabalho baseia-se na determinação das similaridades e diferenças entre plantios de café, definidos através das propriedades levantadas em campo, e sua confirmação ou não, nas imagens dos satélites Ikonos-II e Landsat/ETM+. Foram realizadas análises para determinar a influência das propriedades levantadas em campo, nas diferenças e similaridades espectrais registradas pelas imagens.

Para avaliar os dados das imagens dos dois satélites, foi utilizado o teste de Tukey, para variáveis independentes. Cada talhão foi considerado como uma variável independente, havendo portanto a comparação entre as médias de pares de talhões, com intervalo de confiança de 95\% de probabilidade. Após a análise das médias, em pares de talhões, foram realizados alguns cálculos para avaliar as imagens do Ikonos-II e do Landsat/ ETM+, como: determinação das similaridades e diferenças entre plantios de café, com base nas variáveis agronômicas levantadas em campo, e sua confirmação ou não, nas imagens; determinação da influência das propriedades levantadas em campo, nas diferenças e similaridades espectrais registradas pelas imagens; cálculo da porcentagem de talhões iguais em campo e diferentes nas imagens, iguais nas imagens e diferentes no campo e iguais no campo e nas imagens; e avaliação da correlação existente entre os parâmetros culturais e a resposta espectral do cafeeiro, tendo-se como objetivo o entendimento da relação entre os parâmetros levantados em campo e as respostas espectrais da cultura.

\section{Resultados e Discussão}

Todos os 50 talhões existentes na propriedade foram identificados na imagem do Ikonos-II. Destes, 34 foram localizados de maneira automática na imagem do Landsat/ETM+, correspondentes a $68 \%$ dos talhões existentes. Além do tamanho dos talhões, outra característica que não permitiu a identificação de alguns de- les, na imagem do Landsat/ETM+, foi sua forma geométrica. Os talhões que foram identificados na imagem Ikonos-II e não identificados na imagem Landsat/ETM+, seguidos por suas respectivas áreas (ha), foram: 19, 2; 30, 0,6; 32, 3,5; 33D, 0,6; 43, 0,5; 51, 2,6; 1, 3,1; 3, 2,1; 22, 2; 28, 0,5; 48, 2; 53, 2,5; 6, 1,4; 52, 1,4; 25, 1,1; 27, 0,9.

Para os talhões que não apresentaram diferenças estatísticas significativas entre suas médias de reflectância, nas imagens, verificou-se qual era o porcentual de concordância de cada uma das propriedades levantadas em campo, para os pares de talhões (Tabela 1). O espaçamento foi o parâmetro da cultura que mais influenciou a não-diferenciação estatística dos valores médios da reflectância dos talhões, mesmo tendo sido observadas diferenças nos outros parâmetros de campo.

As porcentagens de pares de talhões diferentes no campo e similares na imagem são dez vezes maiores nas imagens do Landsat do que na imagem pancromática do Ikonos-II (Tabela 2). A porcentagem de pares de talhões diferentes no campo e na imagem é da ordem

Tabela 1. Porcentagem de talhões sem diferença estatística entre suas médias, que estão na mesma classe, em cada uma das quatro variáveis levantadas em campo, por meio de imagens dos satélites Ikonos-II e Landsat/ETM+.

\begin{tabular}{lcccc}
\hline Imagem & Variedade & Espaçamento & Altura & Idade \\
\hline Ikonos -II & & & & \\
Reflectância & 38,8 & 83,3 & 33,3 & 61,1 \\
Valor de nível de cinza & 33,3 & 83,3 & 38,8 & 66,6 \\
\hline Landsat/ETM+ & & & & \\
Banda 3 & 45,6 & 80,4 & 46,7 & 53,2 \\
Banda 4 & 37,5 & 91,6 & 42,7 & 58,3 \\
Banda 5 & 38,2 & 65,4 & 51,8 & 38,3 \\
\hline
\end{tabular}

Tabela 2. Porcentagem de pares de talhões similares no campo ou diferentes, que apresentaram ou não diferença significativa entre suas médias, nas imagens dos satélites Ikonos-II e Landsat/ETM+(1).

\begin{tabular}{lcrcc}
\hline Imagem & $\neq \mathrm{c}=\mathrm{i}$ & $=\mathrm{c} \neq \mathrm{I}$ & $=\mathrm{c}=\mathrm{i}$ & $\neq \mathrm{c} \neq \mathrm{i}$ \\
\hline Ikonos-II & & & & \\
$\quad$ Reflectância & 1,6 & 100,0 & 0,0 & 98,0 \\
$\quad$ Valor de nível de cinza & 1,5 & 98,8 & 1,1 & 98,0 \\
\hline Landsat/ETM+ & & & & \\
$\quad$ Banda 3 & 16,0 & 80,4 & 19,5 & 84,0 \\
Banda 4 & 17,0 & 82,9 & 17,0 & 84,4 \\
Banda 5 & 14,0 & 87,8 & 12,2 & 86,3 \\
\hline
\end{tabular}

(1)Símbolo =c representa pares de talhões que possuem todas as características levantadas em campo iguais; símbolo $\neq c$ representa pares de talhões que possuem pelo menos uma das características levantadas em campo diferente; símbolo =i representa pares de talhões cujas médias não diferem entre si a 95\% de probabilidade; e símbolo $\neq \mathrm{i}$ representa pares de talhões cujas médias diferem entre si a 95\% de probabilidade. 
de 14 a 15 pontos superior na imagem do Ikonos-II, em relação à do Landsat/ETM+. Esses resultados estão diretamente relacionados com a melhoria da resolução espacial e radiométrica do Ikonos-II, em relação ao Landsat/ETM+.

As porcentagens de pares de talhões cujas médias apresentaram diferença estatística significativa a 95\% de probabilidade $(\neq i)$, que são iguais $(=c)$, ou que são diferentes no campo em pelo menos uma das características $(\neq c)$, são muito próximas, independentemente do satélite, da banda e do processamento realizado (Tabela 3). No caso de porcentagens de pares de talhões cujas médias não apresentaram diferença estatística significativa (=i), os valores variaram um pouco mais, mas ainda ficaram próximos.

Nas características dos talhões analisados e na porcentagem de reflectância em cada banda espectral considerada, observa-se uma alta correlação entre os parâmetros espaçamento e idade (Tabela 4). Esta alta correlação positiva já era esperada, uma vez que plantios mais antigos ocupam uma área maior no campo, em virtude de espaçamentos maiores.

Tabela 3. Porcentagem de pares de talhões que apresentaram ou não diferença significativa entre suas médias, nas imagens dos satélites Ikonos-II e Landsat/ETM+, e que são similares ou diferentes no campo ${ }^{(1)}$.

\begin{tabular}{lrrrr}
\hline Imagem & $=\mathrm{i} \neq \mathrm{c}$ & $\neq \mathrm{i}=\mathrm{c}$ & $=\mathrm{i}=\mathrm{c}$ & $\neq \mathrm{i} \neq \mathrm{c}$ \\
\hline Ikonos-II & & & & \\
$\quad$ Reflectância & 100,0 & 7,2 & 0,0 & 92,8 \\
$\quad$ Valor de nível de cinza & 94,4 & 7,1 & 5,6 & 92,9 \\
\hline Landsat/ETM+ & & & & \\
$\quad$ Banda 3 & 91,3 & 7,0 & 8,7 & 93,0 \\
Banda 4 & 92,7 & 7,3 & 7,3 & 92,7 \\
Banda 5 & 93,8 & 7,5 & 6,2 & 92,5 \\
\hline
\end{tabular}

(1)Símbolo $=$ c representa pares de talhões que possuem todas as características levantadas em campo iguais; símbolo $\neq \mathrm{c}$ representa pares de talhões que possuem pelo menos uma das característica levantadas em campo diferente; símbolo =i representa pares de talhões cujas médias não diferem entre si a 95\% de probabilidade; e símbolo $\neq i$ representa pares de talhões cujas médias diferem entre si a 95\% de probabilidade.

Tabela 4. Correlação entre os parâmetros da cultura e a porcentagem de reflectância, para as imagens dos satélites Ikonos-II e Landsat/ETM+, sobre campos de café.

\begin{tabular}{lccccccc}
\hline Parâmetro & $\begin{array}{c}\text { Espaça- } \\
\text { mento }\left(\mathrm{m}^{2}\right)\end{array}$ & $\begin{array}{c}\text { Altura } \\
(\mathrm{m})\end{array}$ & $\begin{array}{c}\text { Idade } \\
(\mathrm{anos})\end{array}$ & $\begin{array}{c}\mathrm{B} 3 \\
(\%)\end{array}$ & $\begin{array}{c}\mathrm{B} 4 \\
(\%)\end{array}$ & $\begin{array}{c}\text { B5 } \\
(\%)\end{array}$ & $\begin{array}{c}\text { Ikonos } \\
(\%)\end{array}$ \\
\hline Espaçamento $\left(\mathrm{m}^{2}\right)$ & 1 & & & & & & \\
Altura (m) & 0,26 & 1 & & & & & \\
Idade (anos) & $0,80^{*}$ & $0,61^{*}$ & 1 & & & & \\
B3 (\%) & $-0,17$ & $-0,56^{*}$ & $-0,32$ & 1 & & & \\
B4 (\%) & $-0,13$ & $-0,15$ & $-0,18$ & $-0,48$ & 1 & & \\
B5 (\%) & 0,03 & $-0,26$ & 0,07 & 0,62 & $-0,06$ & 1 & \\
Ikonos (\%) & $-0,14$ & 0,03 & $-0,15$ & $-0,73$ & $0,81^{*}$ & $-0,48$ & 1 \\
\hline
\end{tabular}

*Significativo a 5\% de probabilidade, pelo teste de Tukey.
A correlação positiva entre a altura e idade da planta mostra que plantas mais altas são mais velhas (Tabela 4). Isto, no entanto, não é regra na cultura do café, por causa do número de podas realizadas nas lavouras.

A altura foi o único parâmetro que mostrou correlação significativa com uma das imagens analisadas (Tabela 4). A correlação negativa da altura com a banda 3 do Landsat/ETM+ pode estar relacionada com a maior quantidade de fitomassa (maior absorção) e com o sombreamento da cultura, o que acarretou menor reflectância da cultura nesta banda. Esta correlação assemelha-se à encontrada por Leonardi (1990).

Foi verificada correlação entre as bandas 3, 4 e 5 do Landsat/ETM+ e o canal pancromático do Ikonos-II (Tabela 4), o que é positivo, do ponto de vista da aplicação de dados de satélite de média resolução espacial.

\section{Conclusões}

1. A imagem pancromática do satélite Ikonos-II possui melhor desempenho na identificação de talhões com características diferentes, em campo, do que a do satélite Landsat/ETM+.

2. Nenhuma das imagens analisadas permite a identificação de talhões com características iguais, em campo.

3. As correções atmosférica e radiométrica, na imagem do canal pancromático do Ikonos-II, não proporcionam ganho substancial nas análises realizadas.

4. A utilização da imagem pancromática do satélite Ikonos-II proporciona a identificação de 1,5 vez mais talhões do que a do satélite Landsat/ETM+.

5. A correlação significativa da reflectância nas bandas 3, 4 e 5 do Landsat/ETM+, com a reflectância do canal pancromático do Ikonos-II, indica que é possível relacionar as imagens dos dois sensores.

\section{Referências}

BATISTA, G.T.; TARDIN, A.T.; CHEN, S.C.; DALLEMAND, J.F. Avaliação de produtos HRV/SPOT e TM/LANDSAT na discriminação de culturas. Pesquisa Agropecuária Brasileira, v.25, p.379-386, 1990.

COLOMBO, R.; BELLINGERI, D.; FASOLINI, D.; MARINO, C.M. Retrieval of leaf area index in different vegetation types using high resolution satellite data. Remote Sensing of Environment, v.86, p.120-131, 2003.

DALLEMAND, J.F.; TARDIN, A.T.; BATISTA, G.T. Sensoriamento remoto e agricultura. Ciência Hoje, v.8, p.44-51, 1988. 
DECHKA, J.A.; FRANKLIN, S.E.; WATMOUGH, M.D.; BENNETT, R.P.; INGSTRUP, D.W. Classification of wetland habitat and vegetation communities using multi-temporal Ikonos imagery in Southern Saskatchewan. Canadian Journal of Remote Sensing, v.28, p.679-685, 2002.

EPIPHANIO, J.C.N.; GLERIANI, J.M.; FORMAGGIO, A.R.; RUDORFF, B.F.T. Índices de vegetação no sensoriamento remoto da cultura do feijão. Pesquisa Agropecuária Brasileira, v.31, p.445454, 1996.

EPIPHANIO, J.C.N.; LEONARDI, L.; FORMAGGIO, A.R. Relações entre parâmetros culturais e resposta espectral de cafezais. Pesquisa Agropecuária Brasileira, v.29, p.439-447, 1994.

FANG, H.L.; WU, B.F.; LIU, H.Y.; HUANG, X. Using NOAA AVHRR and Landsat TM to estimate rice area year-by-year. International Journal of Remote Sensing, v.19, p.521-525, 1998.

GONZALEZ-ALONSO, F.; CUEVAS, J.M.; ARBIOL, R.; BAULIES, $X$. Remote sensing and agricultural statistics: crop area estimation in Northeastern Spain through diachronic Landsat TM and ground sample data. International Journal of Remote Sensing, v.18, p.467-470, 1997.

HAMAR, D.; FERENCZ, C.; LICHTENBERGER, J.; TARCSAI, G.; FERENCZ-ÁRKOS, I. Field estimation for corn and wheat in the Hungarian Great Plain using satellite remote sensing data. Novenytermeles, v.44, p.147-160, 1995

HEROLD, M.; GOLDSTEIN, N.C.; CLARKE, K.C. The spatiotemporal form of urban growth: measurement, analysis and modeling. Remote Sensing of Environment, v.86, p.286-302, 2003.

IPPOLITI-RAMILO, G.A.; EPIPHANIO, J.C.N.; SHIMABUKURO, Y.E. Landsat-5 thematic mapper data for preplanting crop area evaluation in tropical countries. International Journal of Remote Sensing, v.24, p.1521-1534, 2003.

IPPOLITI-RAMILO, G.A.; EPIPHANIO, J.C.N.; SHIMABUKURO, Y.E.; FORMAGGIO, A.R. Sensoriamento remoto orbital como meio auxiliar na previsão de safras. Agricultura em São Paulo, v.46, p.89-101, 1999.

JOHNSON, L.F. Temporal stability of an NDVI-LAI relationship in a Napa Valley vineyard. Australian Journal of Grape and Wine Research, v.9, p.96-101, 2003.

JOHNSON, L.F.; ROCZEN, D.E.; YOUKHANA, S.K.; NEMANI, R.R.; BOSCH, D.F. Mapping vineyard leaf area with multispectral satellite imagery. Computers and Electronics in Agriculture, v.38, p.33-44, 2003.

LEE, D.S.; SHAN, J.; BETHEL, J.S. Class-guided building extraction from Ikonos imagery. Photogrammetric Engineering and Remote Sensing, v.69, p.143-150, 2003.

LEONARDI, L. Influência de parâmetros culturais de cafezais sobre os dados TM/LANDSAT-5. 1990. 171p. Dissertação
(Mestrado) - Instituto Nacional de Pesquisas Espaciais, São José dos Campos.

LIU, W.T.H.; LIU, B.W.Y. Comparação entre três modelos de previsão de safra de café no Estado de Minas Gerais. Ciência e Cultura, v.40, p.801-807, 1988.

LOBELL, D.B.; ASNER, G.P. Comparison of Earth Observing-1 ALI and Landsat ETM+ for crop identification and yield prediction in Mexico. IEEE Transactions on Geoscience and Remote Sensing, v.41, p.1277-1282, 2003.

LUNETTA, R.S.; EDIRIWICKREMA, J.; JOHNSON, D.M.; LYON, J.G.; McKERROW, A. Impacts of vegetation dynamics on the identification of land cover change in a biologically complex community in North Carolina, USA. Remote Sensing of Environment, v.82, p.258-270, 2002.

MOREIRA, M.A. Expansão direta para estimar áreas de soja e milho através de dados multiespectrais e temporais do Landsat-TM. Pesquisas Agropecuária Brasileira, v.26, p.1607-1613, 1991.

PICINI, A.G.; CAMARGO, M.B.P. de; ORTOLANI, A.A.; FAZUOLI, L.C.; GALLO, P.B. Desenvolvimento e teste de modelos agrometeorológicos para a estimativa de produtividade do cafeeiro. Bragantia, v.58, p.157-170, 1999.

READ, J.M.; CLARK, D.B.; VENTICINQUE, E.M.; MOREIRA, M.P. Application of merged 1-m and 4-m resolution satellite data to research and management in tropical forests. Journal of Applied Ecology, v.40, p.592-600, 2003.

SONG, C.; WOODCOCK, C.E. The spatial manifestation of forest succession in optical imagery: the potential of multiresolution imagery. Remote Sensing of Environment, v.82, p.271-284, 2002.

SUGUMARAN, R.; ZERR, D.; PRATO, T. Improved urban land cover mapping using multi-temporal IKONOS images for local government planning. Canadian Journal of Remote Sensing, v.28, p.90-95, 2002.

TANRÉ, D.; DEROO, C.; DUHAUT, P.; HERMAN, M.; MORCRETTE, J.J.; PERBOS, J.; DESCHAMPS, P.Y. Description of a computer code to simulate the satellite signal in the solar spectrum: the 5S code. International Journal of Remote Sensing, v.11, p.659-668, 1990.

VICENTE, J.R.; CASER, D.V.; CAMARGO, A.M.M.P.; OLIVETTI, M.P.A.; PIVA, L.H.O. Comparações entre dados dos censos agropecuários e estimativas das safras agrícolas do Estado de São Paulo. Agricultura em São Paulo, v.37, p.97-104, 1990.

ZULLO JUNIOR, J. Correção atmosférica de imagens de satélite e aplicações. 1994. 190p. Tese (Doutorado) - Universidade Estadual de Campinas, Campinas. 